\title{
EXTRAPOLATION OF LOAD HISTORIES AND SPECTRA
}

\author{
Pär Johannesson \\ Fraunhofer-Chalmers Centre \\ Chalmers Science Park, SE-412 88 Göteborg, SWEDEN \\ Par.Johannesson@fcc.chalmers.se
}

\begin{abstract}
In fatigue life assessments both the material properties and the load characteristics are essential parameters. The life of a component can be experimentally found by performing fatigue tests. In order to get reliable predictions of the life in service, the tests should be performed using variable amplitude loadings that are representative for the service loads. This paper concentrates on the problem of extrapolating a measured load history to a longer time period, for example to a full design life. Using statistical extreme value theory, a new method for extrapolating a time sequence is presented. The obtained extrapolated load spectrum is compared to the result using a method for extrapolating the rainflow matrix.
\end{abstract}

\section{Introduction}

The service life of a component depends on both the load conditions and the fatigue strength. Hence, in order to get a proper fatigue design it is important to consider real customer loads. Many engineering methods are based on finding the worst case scenario, where worst often should be interpreted as a certain severe customer; see e.g. Grubisic [3], Klätschke \& Schütz [5], Socie [7]. These procedures involve extrapolation of measured loads to longer periods of time, typically to a design life. Such an extrapolation should allow more extreme loads than the observed ones. In Socie [7] a statistical method is used that was proposed by Dressler et al. [2], where the rainflow matrix is extrapolated using kernel smoothing.

The main topic of this paper is a method, based on statistical extreme value theory, for extrapolation of a measured time signal to a longer time period, allowing for more extreme cycles than the largest observed ones. The method can be applied to any signal and any purpose, e.g. the extrapolated time signal could be the input to a fatigue test, or the load input to a FEM fatigue analysis. The method will first be explained on a very short signal, where it is easier to see how the method works. It will then be applied to two load histories, one from a train, and one from a car. Further, the obtained load spectrum from the extrapolated time signal will be compared to the load spectrum obtained from the more direct method of computing the extrapolated rainflow matrix.

\section{Method for Extrapolation of a Load History}

A measured signal often represents only a very short part of the design life. When performing variable amplitude tests it is customary to use a measured load history, and repeat this load block until failure. This has the drawback that only the cycles in the measured signal will appear in the extrapolation, even though also other cycles are possible. Especially, this can be critical for the most damaging large amplitude cycles. The methodology here will be to repeat the measured load block, but modify the largest maxima and lowest minima in each block. The random regeneration of each block is based on 
extreme value theory. An example showing the principle of the method is shown in Fig. 1, where three repetitions of a measured block is compared to three extrapolated blocks. Note that only the maxima above a high load level $u_{\max }$, and the minima below a low load level $u_{\text {min }}$, are randomly regenerated. Also note that the extrapolation is performed on the turning points of the signal and not on the originally measured signal.

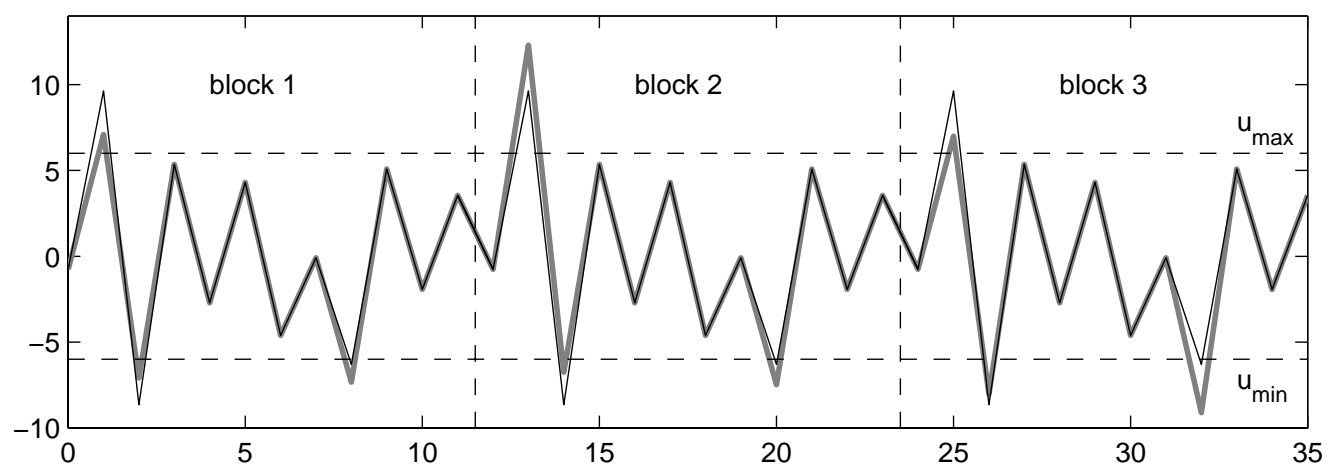

FIGURE 1. Three repetitions of a measured block (black), compared to three extrapolated blocks (grey). The horizontal dashed lines represent the threshold levels, $u_{\min }=-6$ and

$$
u_{\max }=6 \text {, where the extrapolation starts. }
$$

\section{Peak Over Threshold Analysis}

The theoretical base for the method is the so-called Peak Over Threshold (POT) technique in statistical extreme value theory, see e.g. Davison \& Smith [1]. We study exceedances above a level $u$, i.e. the height of the excursions above $u$, see Fig. 2 . If we fix a high load level and study the exceedances above this level, then under certain conditions these exceedances approximately follow an exponential distribution for high enough threshold levels $u$. We then have the approximation for the exceedance $Z=\operatorname{Max}-u \in \operatorname{Exp}(m)$, with cumulative distribution function

$$
F(z)=1-\exp \left(-\frac{z}{m}\right), \quad m=\text { "mean exceedanceabove } u \text { " }
$$

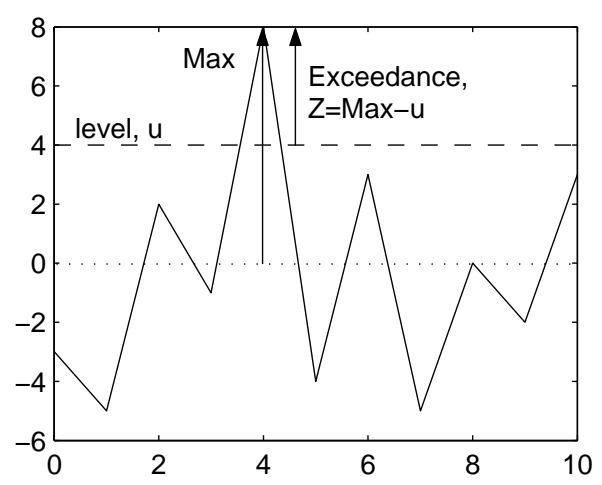

FIGURE 2. Definition of exceedances above a threshold $u$. 
The estimation of the parameter in the exponential distribution is the sample mean of the exceedances $z_{1}, \ldots, z_{N}$

$$
\hat{m}=\frac{1}{N} \sum_{i=1}^{N} z_{i}
$$

As a matter of fact, the exponential distribution of exceedances is equivalent to modelling the global maximum as a Gumbel distribution, which works well in many applications.

\section{Extrapolation of a Load History}

The extrapolation method has been verified on several types of load measurements from different applications. In order to demonstrate the extrapolation two signals have been chosen:

a) a service load on a train from Bombardier Transportation; measurements of stress at a location just above the boogie, and

b) a test track measurement on a car from PSA Peugeot Citroën; vertical forces on the front left wheel.

The input to the method is the turning points of the measurement that have been rainflow filtered to remove small oscillations. In the first example we have extrapolated to a 10 times as long distance, and compare the load spectra of the extrapolations to the measured ones, see Fig. 3. We observe that the extrapolation looks reasonable since it agrees well with the observed load spectrum. Further, the load spectra are extrapolated to higher amplitudes, and is smoother compared to the observed ones. This was exactly the properties we aimed for.
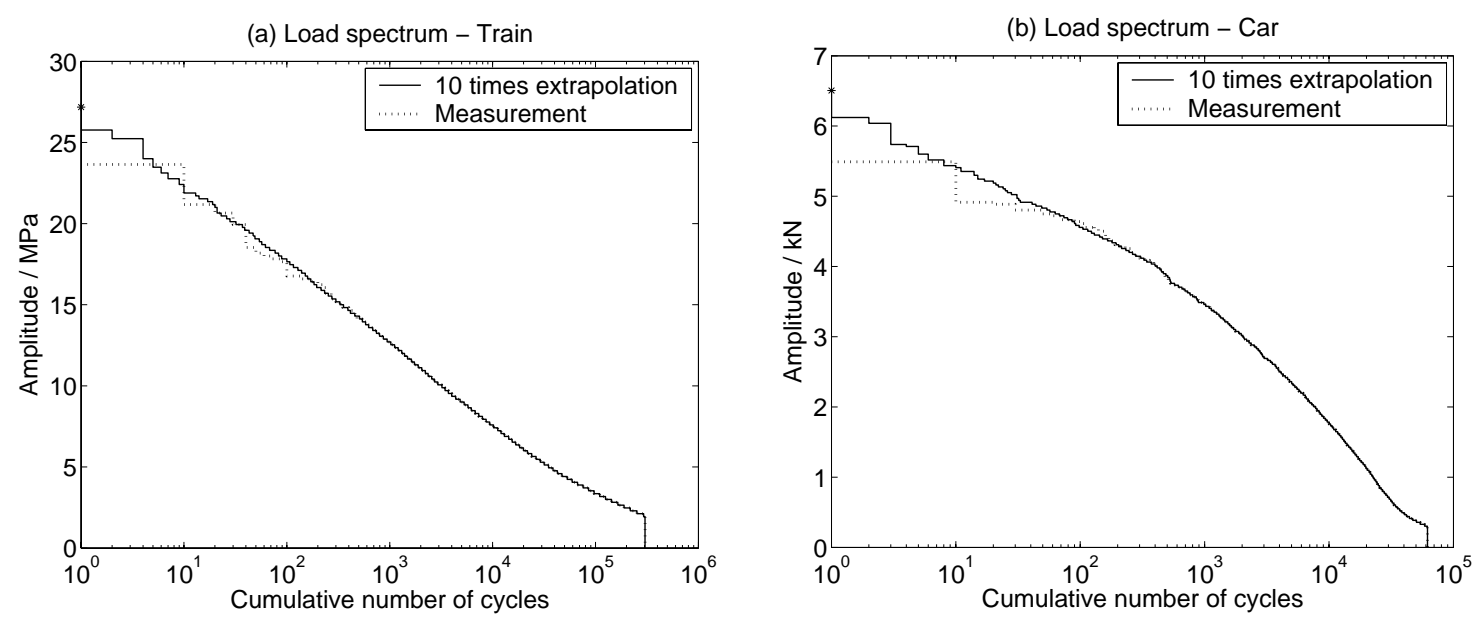

FIGURE 3. Ten times extrapolated load spectra, compared to measure load spectra.

The extrapolation method is based on a random simulation of the high maxima and low minima. Hence, the resulting extrapolated turning points, and hence also the load spectra, will be different for each new simulation. This scatter between different simulations is illustrated in Fig. 4, where 10 simulations of 10 times extrapolations are shown. The 10 times extrapolations are compared to the 100 times extrapolation (obtained from the $10 \times 10$ times extrapolations). We observe that the scatter increases for higher amplitudes, and that the 100 times extrapolation lies in the middle. 
(a) Load spectrum - Train

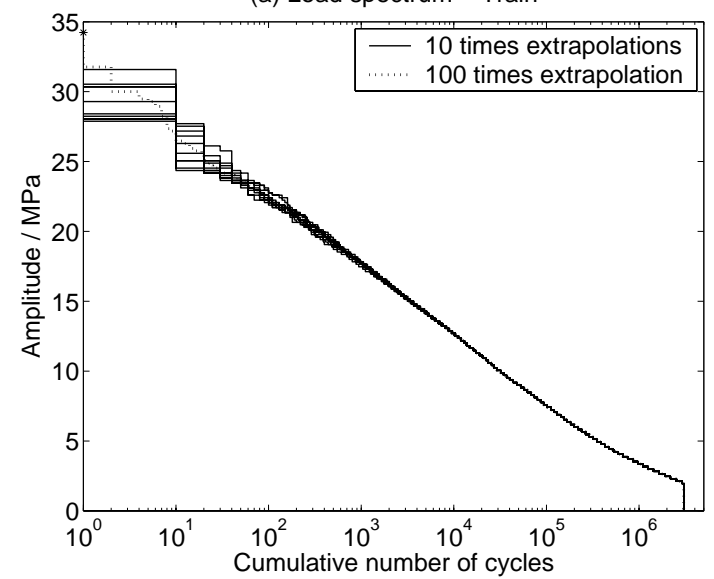

(b) Load spectrum - Car

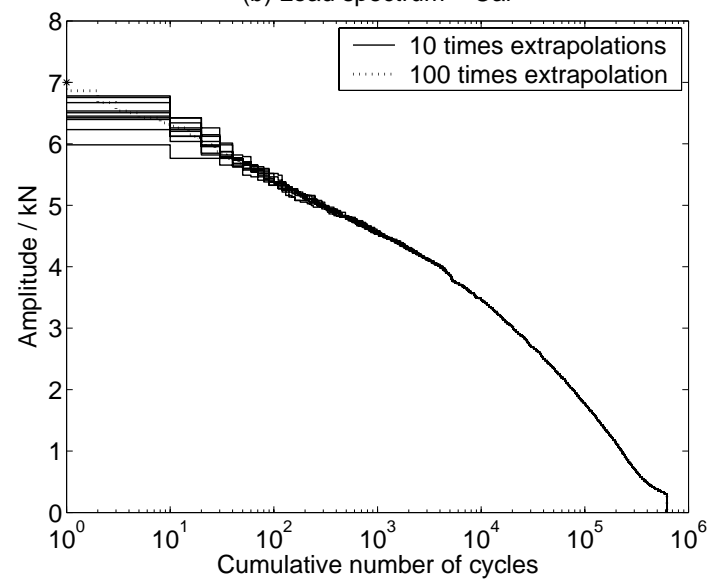

FIGURE 4. Ten load spectra for ten times extrapolation, and the load spectrum for 100 times extrapolation.

\section{Choice of Threshold Levels}

For the extrapolation we need to choose the threshold level where the extrapolation shall start, see Fig. 1. The choice of proper threshold levels is a difficult problem that demands some judgements. Note that the levels need to be chosen high enough for the extreme value theory to be a reasonable approximation, however also low enough to get a sufficient number of exceedances. Hence, it is important to verify that the exponential assumptions are fulfilled for the threshold levels in question. In Fig. 5 the exceedances above $12 \mathrm{MPa}$ and the exceedances below -13 MPa are plotted for the train load. The agreement with the exponential assumption can be evaluated in the exponential probability plot, where the observations should approximately follow the straight line, and in Fig. 5 the agreement is good.
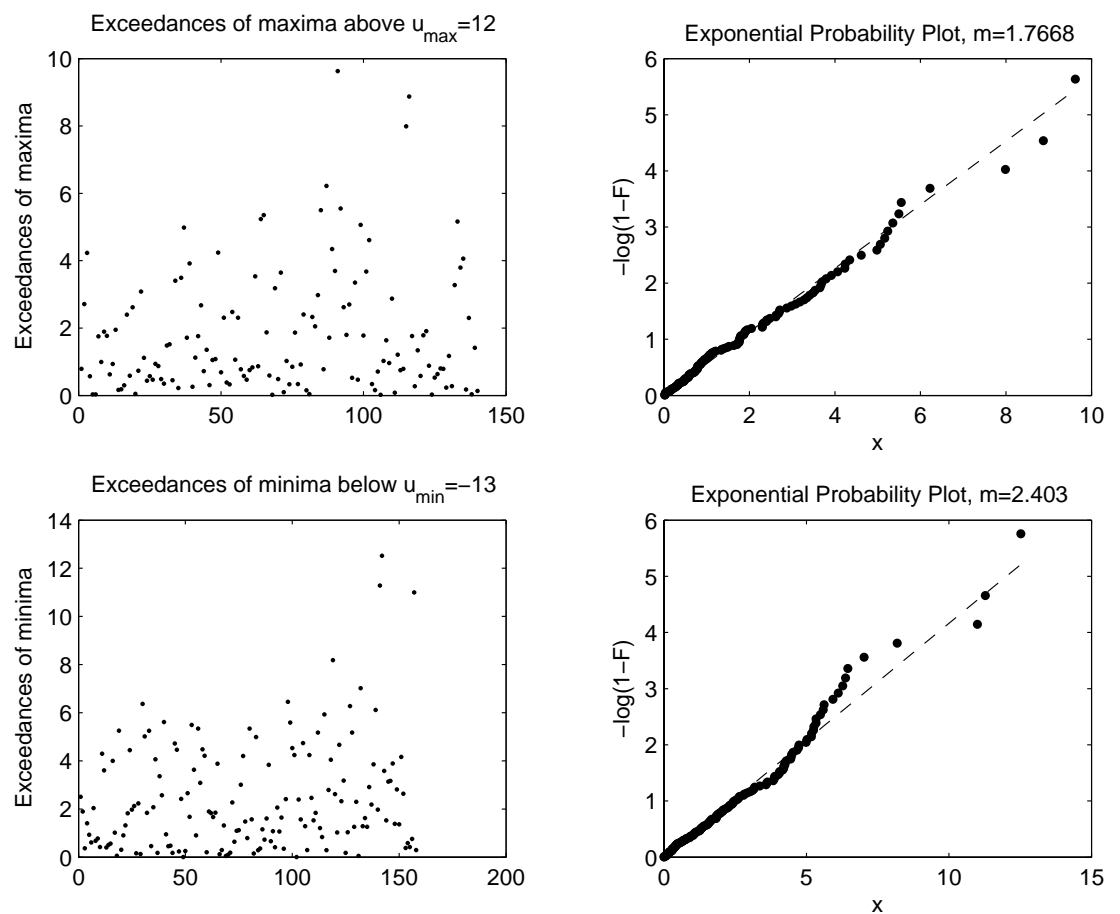
FIGURE 5. Exceedances for train load.

The choice of proper threshold levels is the most tricky part of the extrapolation. Here one needs to use experience together with the diagnostic tools to ensure that the exponential distribution is a good approximation. A default choice that seems to work well is to find threshold levels such that there are about $\sqrt{N_{0}}$ exceedances, where $N_{0}$ is the number of cycles in the signal.

\section{Extrapolation - Time Domain vs. Rainflow Domain}

The method described in this paper is an extrapolation in the time domain. An alternative method is to extrapolate in the rainflow cycle domain, using the method in Johannesson \& Thomas [4]. The two methods for extrapolation are both based on the theory of statistical extreme values. Hence, the resulting extrapolated load spectra should be similar. However, the time domain method results in an extrapolated time signal where the order of the cycles is preserved, while the rainflow domain method gives an extrapolated rainflow matrix. More precisely, the time domain method simulates extrapolated turning points, and then calculates the extrapolated rainflow matrix from this signal. Hence, for the time domain method, the result is an $N$ times extrapolated load, while for the rainflow domain method the result is a limiting rainflow matrix representing $N=\infty$ number of repetitions. In Fig. 6 the load spectra from the original measurements, from 100 times time domain extrapolation, and from rainflow extrapolation, are shown. Both types of extrapolations give similar results. However, the rainflow domain method is well adopted for really long extrapolations of the load spectrum, whereas the time domain method in those cases will be computationally less tractable. Also note that the maximum load amplitude is extrapolated. The train load measurement has maximum amplitude of $27 \mathrm{MPa}$, and the extrapolation about $32 \mathrm{MPa}$. The corresponding maximum values for the car load is $5.5 \mathrm{kN}$ and $7 \mathrm{kN}$. Hence, in a fatigue test for the car application, instead of repeating the amplitude $5.5 \mathrm{kN} 100$ times, the extrapolation applies one amplitude of $7 \mathrm{kN}$ and several smaller. This is our informed guess of what would be the load spectrum if we had a 100 times longer measurement.

(a) Load spectrum - Train

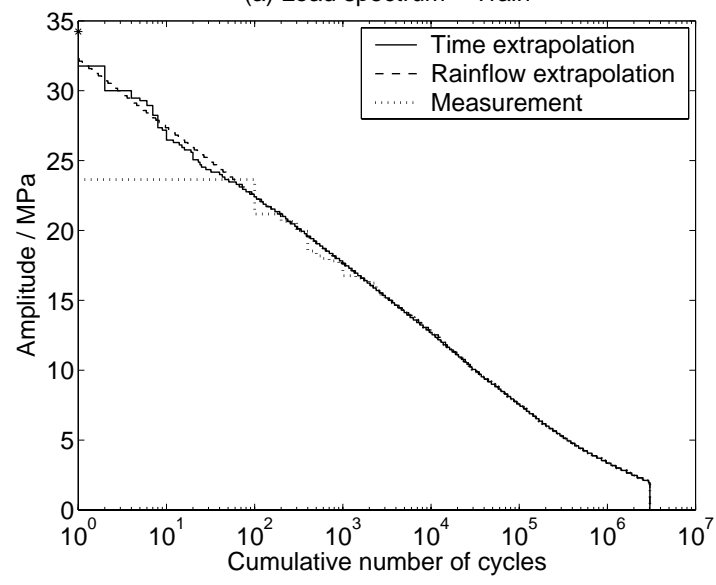

(b) Load spectrum - Car

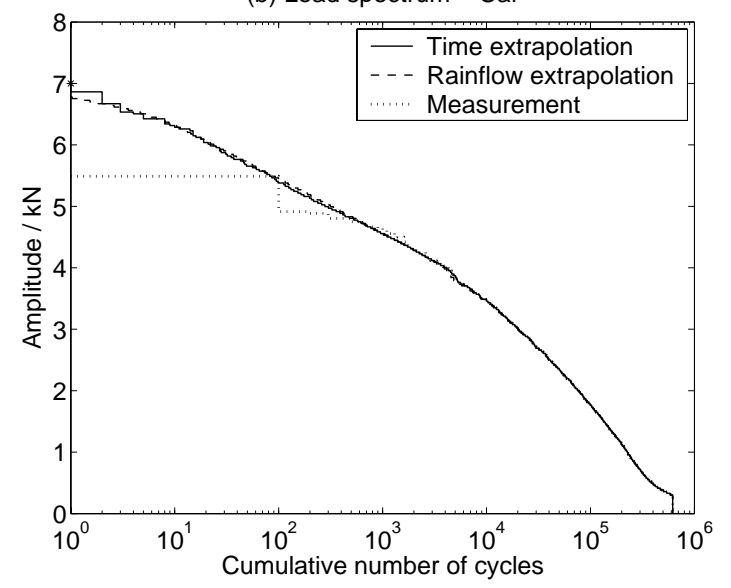

FIGURE 6. 100 times extrapolation, comparing time and rainflow domain methods. 


\section{Conclusions \& Discussion}

The method presented for extrapolating a load signal to a longer period of time has proved to work well for the two examined measurements. The main use of the method should be for generation of time signals that are aimed for fatigue tests, or as load input to fatigue life assessments.

If our main goal is to generate a longer load block from a measured one, an obvious advantage of extrapolating in the time domain is that we directly get a time signal. In the rainflow domain we get a limiting rainflow matrix from which we need to generate a time signal, e.g. using the Markov method described in Rychlik [6], where the rainflow matrix is inverted into a Markov chain model, from which it is easy to simulate a time signal of arbitrary length. Another advantage of the time domain method could be that the generated sequence of cycles is realistic since it is directly based on a measured sequence.

If our goal is to extrapolate the load spectrum, the method of rainflow extrapolation has the properties of being more computationally efficient, and estimates the shape of the load spectrum for an infinitely long measurement. However, the time domain method is more robust, since it only uses the extreme value theory for extrapolating to extreme load levels, while the rainflow domain method uses an additional extreme value approximation for the shape of the matrix.

\section{Acknowledgements}

The author thanks Bombardier Transportation and PSA Peugeot Citroën for their support, and for permission to use their load data in the examples.

\section{References}

1. Davison, A. C., and Smith, R. L., Journal of Royal Statistical Society, Ser. B, vol. 52, 393-442, 1990.

2. Dressler, K., Gründer, B., Hack, M., and Köttgen, V.B., SAE Technical Paper 960569, 1996.

3. Grubisic, V., International Journal of Vehicle Design, vol. 15, 8-26, 1994.

4. Johannesson, P. and Thomas, J.-J., Extremes, vol. 4, 241-262, 2001.

5. Klätschke, H., and Schütz, D., Materialwissenschaft und Werkstofftechnik, vol. 26, 404415, 1995.

6. Rychlik, I., International Journal of Fatigue, vol. 18, 429-438, 1996.

7. Socie, D., International Journal of Materials and Product Technology, vol. 16, 295-303, 2001. 